\title{
The Evolution and Prevalence of Knee Injuries: Repair at What Cost?
}

\author{
RAChel Spanier \\ University of Victoria \\ rspanier@uvic.ca
}

\begin{abstract}
Knee injuries are a common nuisance in the lives of many active individuals. They can stem from tissue damage, ligament damage or bone and/or cartilage damage. Through the exploration of major knee injuries, prevention and more efficient rehabilitation can be attained. The most common knee injuries are analyzed and each of their main cause is examined in this paper. Orthopaedic surgery techniques are discussed, such as, $A C L$ reconstruction, meniscal resection/meniscectomy and high tibial osteotomy. These surgical techniques have evolved immensely in response to the high occurrence of serious knee injuries in active individuals. Many of these injuries originate from too much torque and/or torsion on the knee joint occurring during sports. The faulty anatomy of the knee joint and the glacial pace of evolution play a much larger role than just prevalence of injuries. The economic cost of major knee injury repairs is far too high in private health care systems, which in turn results in a poorer quality of psychosocial health in those injured and unable to receive the corrective surgeries.
\end{abstract}

Keywords: knee injury; knee anatomy; knee joint; surgery; ligament injury; meniscal injury; meniscal repair; meniscectomy; high tibial osteotomy; sports injury; rehabilitation

\footnotetext{
"I would like to give a special thanks to Emily Arvay - my English professor. Without her encouragement and support, this article would not have been possible.
} 


\section{INTRODUCTION}

$\mathrm{K}$

NEE INJURIES are a common nuisance in the lives of many elite athletes. Injury to a major joint, such as the knee, can be substantial. Knee injuries can stem from tissue damage, ligament (band of fibrous connective tissue that serves to connect bones) damage, and bone or cartilage damage. This paper will examine the anatomy of the knee joint and major knee injuries. The prevalence of knee injuries is demonstrated through statistics and an analysis of high-risk populations. Orthopaedic surgery techniques such as ACL reconstruction, meniscal repair/resection, and high tibial osteotomy will be discussed.

Hopper and Grainger (2010) suggest that knee injuries are so widespread because physical activity and participation of sports are more readily accepted by the general population as a part of everyday life - more individuals engage in such activities, at higher intensity levels, and at a higher frequency than ever before. Most of these injuries result from too much torque and torsion on the knee joint, which has not evolved in sync with the demands of physical activity. Due to this imbalance, major knee injuries occur in greater numbers - in the US, over 200,000 ACL tears are reported annually, with over half being reconstructed. Advanced surgical techniques coupled with increased awareness can potentially prevent undesirable and extremely painful consequences of a knee injury. In situations where the injury is not prevented, accessibility to surgical and rehabilitative resources should be readily available. However, in private health care systems and undeveloped nations, the cost of repair is too high. The economic cost of repairing knee injuries impedes the quality of life in patients. Therefore, the anatomy of the knee and glacial pace of evolution become much more important in the overall quality of life in active individuals. The failings of the knee joint and the financial cost of repair have a direct effect on the psychosocial health of the injured in private health care systems. In public health care systems, repairing major knee injuries can lead to greater gain in the economy by reducing the period that an individual is injured and unable to work 
and by removing the negative factors of injury that can impede the quality of life.

\section{ANATOMY}

The knee joint consists of the tibiofemoral joint (which connects the two main bones in the leg - the superior femur and the inferior tibia) and the patellofemoral joint (which connects the femur and the patella - the "knee cap"). The menisci - two cartilaginous pads separating and reducing friction between the femur and the tibia - and the ligaments keep the joint stable (Hopper \& Grainger, 2010). The menisci are divided into the medial meniscus, which is U-shaped, and the lateral meniscus, which is C-shaped; both consist of fibrous cartilage (Hopper \& Grainger, 2010). They function as shock absorbers for impact on the knee joint. Only one third of the menisci retain vascularity by adulthood, creating a challenging atmosphere for repair of damaged menisci, as lack of blood supply discourages healing (Hopper \& Grainger, 2010). The cruciate ligaments consist of the anterior cruciate ligament (ACL), which attaches to the anterior portion of the tibia, and the posterior cruciate ligament (PCL), which attaches to the posterior portion of the tibia (Hopper \& Grainger, 2010). The ACL and PCL cross over each other, forming an "X" deep within the knee joint (Atanda Jr, 2012). The $\mathrm{X}$ formation also accounts for the name cruciate as it means "crossing over". Together, they help keep the knee joint stable and prevent the tibia from moving too far forward (Atanda Jr, 2012). Both cruciate ligaments can be seen in Figure 1. The collateral ligaments include the medial collateral ligament (MCL) and the lateral collateral ligament (LCL), which both function as stabilizing elements of the knee joint (Hopper \& Grainger, 2010). The term "collateral" in anatomy is defined as a subordinate or an accessory part. The MCL prevents the knee joint from buckling inwards, while the LCL prevents the knee joint from buckling outwards (Brown Education, 2004). 
Figure 1: Head of right tibia seen from above, showing menisci and attachments of ligaments. Image: Gray's Anatomy (public domain)

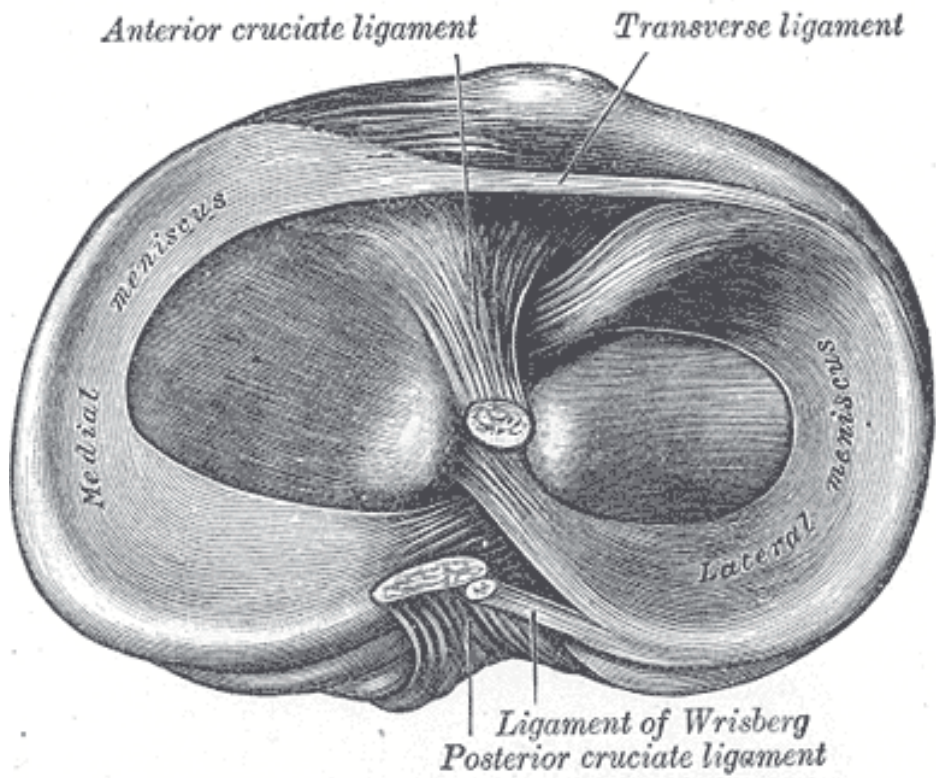




\section{COMMON INJURIES}

Knee injuries account for one quarter of all sporting injuries and take the longest time and effort to heal (Brown Education, 2004). The most common knee injuries are torn or severed ligaments (both cruciate and collateral) and meniscal tears (Fadale \& Hulstyn, 2000). Ligaments tears can be caused by contact, such as a football tackle or non-contact injuries, such as coming to a quick stop combined with a directional change (Brown Education, 2004). The combination of stopping quickly and changing direction simultaneously is the motion that most commonly induces ligament tears.

Meniscal tears occur due to a combination of stopping quickly and changing direction simultaneously. Meniscal tears are more problematic than ligaments tears as there is a lack of blood supply for healing (Kurzweil \& Friedman, 2002). Medial meniscus tears are more common than lateral meniscus tears because the medial meniscus is attached to the MCL, making it more robust, less flexible, and less adaptable to rotation (Hopper \& Grainger, 2012). For most elite athletes, tears in the ligaments and menisci of the knee joint require corrective surgery to allow for full rehabilitation (Brown Education, 2004).

\section{Ligament tears}

Collateral ligament tears rarely require surgical intervention as they receive sufficient blood supply to promote optimal healing (Lee et al., 2014). For collateral ligaments that are severely torn in the middle of the ligament, the torn ends of the ligament are sewn back together. For collateral ligaments that are torn at the point of connection with the femur or tibia, the collateral ligament can be sewn back to the point of connection using large sutures or reattached through the use of a bone staple or metal screw. Cruciate ligament tears require surgical intervention ninety to one hundred percent of the time; for severe tears of the ACL and PCL, reconstruction is necessary (Brown Education, 2004). ACL and PCL reconstruction involve harvesting an allograft - a portion retracted from a specific type of cells such as, the 
hamstring tendon or patellar tendon, then using the harvested graft to replace the severed ligament (Atanda Jr, 2012). After ACL/PCL reconstruction, there is an extended period of rehabilitation, which lasts 6-18 months (Brown Education, 2004). Intense physical therapy must be executed promptly after surgery and continued throughout the entire rehabilitation period (Atanda Jr, 2012). It should be noted that following PCL reconstruction, special emphasis is placed on the rehabilitation and strengthening of the quadriceps to protect the healing PCL (Brown Education, 2004).

\section{Meniscal tears}

Meniscal tears require surgery for the majority of cases (Hopper \& Grainger, 2010). If a tear is located in the region of available blood supply, then a meniscal repair is done, which simply involves sewing the torn ends back together (Brown Education, 2004). For tears that do not receive blood supply, a meniscal resection (meniscectomy) is necessary. A meniscectomy involves resecting, or cutting out, the damaged meniscus portion and then smoothing out the remaining portion of the meniscus (Kurzweil \& Friedman, 2002). The recuperation period following a meniscal surgery is $4-6$ weeks, but for elite athletes who devote hours of rehabilitation a day, the period can be as short as 2 weeks (Fadale \& Hulstyn, 2000). However, there are disadvantages of meniscectomies, such as the development of osteoarthritis, which is defined as the degeneration of the bone and cartilage in a joint due to excessive impact (Brown Education, 2004). Osteoarthritis can only be accommodated through a high tibial osteotomy or a full knee replacement (Mauro \& Bradley, 2014). A high tibial osteotomy involves cutting the tibia and creating a wedge in the bone, then filling the wedge with a allograft of bone and fixing it in place with a plate and multiple screws. Through this procedure, the weightbearing axis is shifted onto the healthy part of the knee, which improves the overall function of the joint (Mauro \& Bradley, 2014). 


\section{THE INJURED INDIVIDUAL}

Injuries affect the quality of life of the injured individual. Injuries affect the physical aspect of a human being - major knee injuries can directly affect the ability to participate in sports, daily activities and employment requirements (Louw et al., 2008). A sedentary lifestyle is considered a risk factor for systemic disease, disability, and death (Louw et al., 2008). Knee injuries reduce mobility, which can affect performance in employment settings and can lead to financial stress. Injuries affect the psychological aspect of a human being as well - being injured often takes away an individual's independence: "Hope and social support are very important features in providing psychological help as people face life challenges such as sport injuries" (Lu et al., 2013). Independence plays a critical role in bringing personal meaning and enrichment to an individual's life (Zirkel \& Cantor, 1990). The injured individual must manage the pain of the injury but also manage detrition in other areas of their life due to said injury. Therefore, the quality of life and the psychosocial health of the injured individual are often much lower than that of an individual whom is not injured.

\section{Socioeconomic cost}

In New Zealand, 238,488 knee ligament injuries were reported over a course of 5 years, and eighty percent of all knee ligament injuries required ACL reconstruction surgery (Gianotti, 2009). High-risk populations that are vulnerable to major knee injuries include females and adolescents (Louw et al., 2008), and men between the ages 20-39 and 50-59 expose themselves to higher risk situations regarding potential knee injury than other age groups (Gianotti, 2009). With the knowledge of the injury-inducing motion, it is apparent how easily the knee joint can give out by simply planting the foot the wrong way. Knee injuries are becoming an epidemic and it is clear that the faulty anatomy of the knee cannot be changed by humans and will not be changed by evolution for thousands of years. Therefore, the 
surgical techniques discussed above begin to bear more importance in terms of quality of life of those injured. In most developed nations, knee repair surgeries are quite accessible. In public health care systems, these surgeries tend to have long wait times but they are actively performed upon all those that desire the surgery. However, in nations with private health care, affording the surgery then becomes an issue. The average cost of an ACL reconstruction surgery is $\$ 10,326$ US dollars (Lubowitz \& Appleby, 2011). The median household income in the United States was \$51,017 in 2012 (DeNavas-Walt et al., 2013). The average household will not and cannot afford to spend one fifth of their total annual income on an elective knee repair surgery. Even with health insurance, the patient would still get billed a large portion of the cost. Therefore, in private health care systems, the quality of life of most injured patients is lowered and remains lowered for a prolonged period of time as a result of being unable to afford the corrective knee surgery.

In contrast, in public health care systems nations such as Canada, individuals do not have to spend any money out of pocket to receive corrective knee injury surgeries. Canadians (and those in other public health care systems) have decreased periods of being injured, accelerated periods of recovery, and overall a greater quality of life. Public health care systems provide the correct resources to remove the detrimental factors surrounding an injury without forcing injured individuals to finance one fifth of their total annual salary. Through the process of surgically repairing major knee injuries for no cost out of pocket, society is in turn putting more money back into the economy by reducing the period individuals are injured, unable to work and collecting employment insurance. By increasing the quality of life of injured individuals through surgical repair, the society and the economy is stimulating itself. There are many components to the public versus private health care system debate, however, in terms of repairing major knee injuries and the general well-being of those injured, the public health care system is arguably more successful. In public health systems, the cost of a major knee repair is justified by the reduction in socioeconomic cost. 


\section{CONClusion}

Through the examination of the anatomy of the knee, the most common injuries, and corrective surgical techniques, a major issue is apparent - active individuals are not equipped to deal with the intense physical activity that they put their bodies through. Evolution has not caught up with the demands that active individuals are placing on their knee joints and these joints eventually break down, leading to injuries that require surgical intervention. Until evolution catches up with our desired demands, we can only use these preventative techniques to avoid major injuries to the imperative knee joint. The faulty anatomy of the knee joint and the glacial pace of evolution play a much larger role than just prevalence of injuries. The economic cost of major knee injury repairs is far too high in private health care systems, which in turn results in a poorer quality of psychosocial health in those injured and unable to receive the corrective surgeries. However, in public health systems, repairing major knee injuries can lead to societal and economic growth by reducing injury time and increasing quality of life. 


\section{REFERENCES}

Atanda Jr, A. (2012). Kids Health. Anterior Cruciate Ligament (ACL) Injuries. Retrieved April 10, 2014, http://kidshealth.org/parent/ nutrition_center/exercise_safety/acl_injuries.html

Brown Education. 2004. Knee Injuries and Therapies in Competitive Athletes. Medial and Lateral Collateral Ligaments. Retrieved April 10, 2014, from http://biomed.brown.edu/Courses/BI108/BI10 8_2004_Groups/Group06/Group6project/Therapy/References.htm

DeNavas-Walt, C., Proctor, B.D., \& Smith, J.C. (2013). Income, Poverty, and Health Insurance Coverage in the United States: 2012. Current Population Reports, pp. 60-245.

Fadale, P.D., \& Hulstyn, M.J. (2000). Common athletic knee injuries. Medicine and Health, 83(6), 178.

Gianotti, S.M., Marshall, S.W., Hume, P.A., \& Bunt, L. (2009). Incidence of anterior cruciate ligament injury and other knee ligament injuries: A national population- based study. Fournal of Science and Medicine in Sport / Sports Medicine Australia, 12(6), 622-627. http://dx.doi.org/10.1016/j.jsams.2008.07.005

Hopper M., \& Grainger, A. (2010). Knee Injuries. In P. Robinson (Eds.), Essential Radiology for Sports Medicine. New York: Springer. http://dx.doi.org/10.1007/978-1-4419-5973-7_1

Kurzweil, P.R., \& Friedman, M.J. (2002). Meniscus: Resection, repair, and replacement. Arthoscopy: The fournal of Arthroscopic \& Related Surgery, 18(2), S1, 33-39. http://dx.doi.org/10.1053/jars.2002.31790

Lee, Y.H.D., Siebold, R., \& Paessler, H.H. (2014). Implant-free ACL reconstruction: a review. Archives of Orthopaedic and Trauma Surgery, 134(3), 395-404. http://dx.doi.org/10.1007/s00402-013-1913-4

Louw, Q. A., Manilall, J., \& Grimmer, K. A. (2008). Epidemiology of knee injuries among adolescents: A systematic review. British fournal of Sports Medicine, 42(1), 2-10. http://dx.doi.org/10.1136/bjsm.2007.035360

Lu, F.J.H., \& Hsu, Y. (2013). Injured athletes' rehabilitation beliefs and subjective well-being: The contribution of hope and social support. Journal of Athletic Training, 48(1), 92-98.

Lubowitz, J.H., \& Appleby, D. (2011). Cost-effectiveness analysis of 
the most common orthopaedic surgery procedures: Knee arthroscopy and knee anterior cruciate ligament reconstruction. Arthroscopy: The fournal of Arthroscopic \& Related Surgery: Official Publication of the Arthroscopy Association of North America and the International Arthroscopy Association, 27(10), 1317-1322. hitp///dx.doi.org/10.101 Mauro, F. \& Bradley, N. (2014). High tibial osteotomy. Current Orthopaedic Practice. 25(1), 71-74. http://dx.doi.org/10.1097/bco.0000000000000053 PhysioWorks Web site (n.d.). Retrieved May 23, 2014, from http:// physioworks.com.au/Injuries-Conditions/Regions/ligament-injury Smith, J.O., Wilson, A.J., \& Thomas, N.P. (2013). Osteotomy around the knee: evolution principles and results. Knee Surgery, Sports Traumatology, Arthoscopy, 21(1), 3- 22. http://dx.doi.org/10.1007//00167-012-2206-0 Snyder, J. (2013). ACL reconstruction: When can I play again? Retrieved May 23, 2014, from http://orthopedicmanualpt.com/tag/ physical-therapy/page/2/

Zirkel, S., \& Cantor, N. (1990). Personal construal of life tasks: Those who struggle for independence. Journal Of Personality And Social Psychology, 58(1), 172-185. http://dx.doi.org/10.1037/0022-3514.58.1.172 